\title{
HIGH DIELECTRONIC SATELLITE LINES IN SOLAR \\ FLARE IRON SPECTRA
}

F. Bely-Dubau

Observatoire de Nice, Nice, France

\section{A.H. Gabriel}

ARD Appleton Laboratory, Culham Laboratory, Abingdon, Oxfordshire, Great Britain

S. Volonté

Departement d'Astrophysique, Universite de Mons, 7000 Mons, Belgique

New calculations have been carried out in intermediate coupling of the wavelengths and intensities of the Fe XXIV transitions $1 s^{2} n \ell-1 s 2 p n \ell$ with $n \geq 3$.

It is shown that some of these lines are situated on the short wavelength side of the helium-like Fe XXV resonance transition. For the intensities new calculations of the rates of autoionization and radiative decay have been made for the levels involved. Only dielectronic capture has been considered because the contribution of direct inner-shell excitation of the doubly excited states with $n \geqslant 3$ is expected to be small in low density astrophysical plasmas.

For this reason both wavelengths and intensities are compared with the only available solar flare Iron spectra of Grineva et a1. (1973). Some features not yet identified in these spectra can be explained with the results of the present calculations. These lead to an important new diagnostic capability for solar flare soft $X$-ray spectra. 